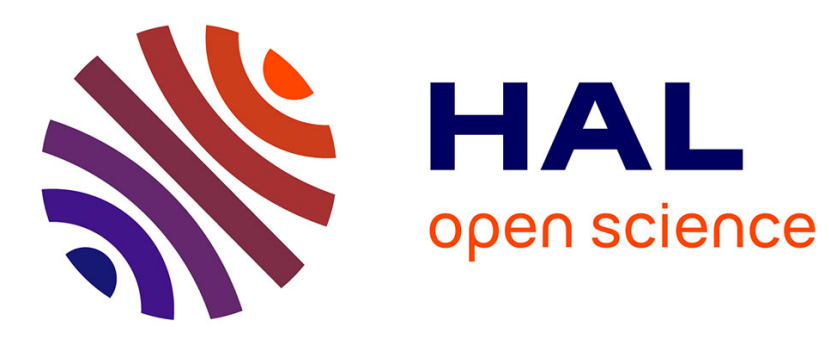

\title{
Wrinkle formations in axi-symmetrically stretched membranes
}

\author{
Jean-Christophe Géminard, Roberto Bernal, Francisco Melo
}

\section{To cite this version:}

Jean-Christophe Géminard, Roberto Bernal, Francisco Melo. Wrinkle formations in axi-symmetrically stretched membranes. European Physical Journal E: Soft matter and biological physics, 2004, 15 (2), pp.117-126. ensl-00180271

\section{HAL Id: ensl-00180271 \\ https://hal-ens-lyon.archives-ouvertes.fr/ensl-00180271}

Submitted on 19 Dec 2007

HAL is a multi-disciplinary open access archive for the deposit and dissemination of scientific research documents, whether they are published or not. The documents may come from teaching and research institutions in France or abroad, or from public or private research centers.
L'archive ouverte pluridisciplinaire HAL, est destinée au dépôt et à la diffusion de documents scientifiques de niveau recherche, publiés ou non, émanant des établissements d'enseignement et de recherche français ou étrangers, des laboratoires publics ou privés. 


\title{
Wrinkle formations in axi-symmetrically stretched membranes
}

\author{
J.-C. Géminard ${ }^{\mathrm{a}}$, R. Bernal, and F. Melo \\ Departamento de Física Universidad de Santiago de Chile and Center for Advanced Interdisciplinary Research in Materials, \\ CIMAT, Avenida Ecuador 3493 - Santiago, Chile
}

Received 26 May 2004 and Received in final form 10 August 2004 /

Published online: 19 October 2004 - (c) EDP Sciences / Società Italiana di Fisica / Springer-Verlag 2004

\begin{abstract}
We study experimentally the main features of wrinkles that form in an initially stretched and flat elastic membrane when subjected to an axi-symmetric traction force at the center. The wavelength and amplitude of the wrinkle pattern are accurately characterized as the membrane tension and the traction forced are varied. We show that wrinkles are the result of a supercritical instability and appear for a welldefined critical traction force that is a function of the membrane tension. Wrinkle length and amplitude increase as the traction force is increased further. By contrast, both quantities decrease as the membrane tension is increased. Calculations based on symmetry arguments and elastic-energy minimization are in good agreement with experiments and provide a simple way to investigate configurations that are difficult to access experimentally. Such problems include wrinkles in elastic nano-films on finite-thickness viscous substrates used in semiconductor technology or in cellular forces detection.
\end{abstract}

PACS. $46.32 .+\mathrm{x}$ Static buckling and instability - 87.19.St Movement and locomotion - 85.40.Ls Metallization, contacts, interconnects; device isolation

\section{Introduction}

Standing films, plates or membranes under longitudinal compressive stress spontaneously buckle to allow for the expansion of the film by bending out of the nominal film plane. In such configurations, buckling produces wrinkles that are perpendicular to the direction of applied compression $[1,2]$. However, less intuitive buckling occurs when an elastic band stripe is subjected to large enough longitudinal stretching in its plane $[3,4]$; the film buckles to relax the in-plane strain incompatibility generated by the Poisson effect. Hence, wrinkles accommodate themselves parallel to the direction of the applied tension. In general, the buckling wavelength is a compromise between the relaxation of in-plane strain and the elastic stresses associated with bending. In practice, the constraint provided by an elastic substrate, to which most films are bounded, can prevent buckling whereas a viscous substrate allows it, but on a time scale set by the viscous flow $[5,6]$.

Wrinkling in elastic films under stress is ubiquitous in nature. Among common systems exhibiting this phenomenon are our skin, textiles and more specifically thin coatings subjected to stresses mismatch $[7,8]$. Apart from a basic interest, wrinkled membranes have found unexpected applications recently. For instance, in modern

\footnotetext{
a Permanent address: Laboratoire de Physique, Ecole Normale Supérieure de Lyon, 64, Allée d'Italie, 69364 Lyon cedex 07, France; e-mail: jean-christophe.geminard@ens-lyon.fr
}

technology, pre-wrinkled conductive films are proposed as stretchable electrical contacts required in large-area electronics [9]. In medicine, surgery has always involved cutting, thickening contraction and in many cases wrinkling of the skin. Potential applications of wrinkle mechanics to improve wound healing have been recently summarized by E. Cerda [10]. In turn, in cell biomechanics, wrinkled patterns produced by cells crawling onto elastic membranes (several nanometers thick), provided a useful tool to test living-cells locomotion. With this method, the length of the wrinkles has been correlated to the force applied by the cell cytoskeleton $[11,12]$. In this article, we present experimental as well as theoretical studies of the wrinkles that form on an initially flat elastic membrane when subjected to an axi-symmetric traction force. In our system such a force is applied over a central region having a given radius by pulling symmetrically the membrane. With this configuration we intend to mimic, for instance, the pattern resulting from the homogeneous contraction of a nearly circular living cell when attached to an elastic film [13] or a circular wound as described recently by E. Cerda [10]. In addition to the central force, the membrane can be stretched radially to vary its tension. Our results show that the length of the wrinkles as well as their amplitude increase as the traction force is increased beyond a well-defined critical value which is a function of the membrane tension. By contrast, both quantities decrease as the initial membrane tension is increased. We show thus that wrinkles appear as a consequence of a 
supercritical instability. Beyond the instability threshold, the number of nucleated wrinkles is nearly independent of the central force, varies very slowly with membrane tension, and is a linear function of the size of the central object. In addition, if the tension vanishes, our results predict that wrinkles develop in the whole membrane. These observations challenge the validity of previous membrane force measurements [12], due to living-cell traction, based on the wrinkle length. We discuss under what conditions these measurements are reliable and we propose alternative approaches. To account for our experimental results, we develop a theoretical approach, based on bending- and stretching-energy minimization, that capture well the features described above. Symmetry arguments allow us to solve the complex membrane equations with simple numerical calculation.

Our article is organized as follows. Section 2 is devoted to the description of the procedure used to deform the membrane and to introduce the minimal ingredients necessary to explain why the membrane buckles. In addition, by developing simple equilibrium forces considerations and by neglecting the bucking threshold, we recover recent experimental and theoretical results [10] obtained in the limit of an infinitely thin and infinitely large membrane under stress. In our experiment, the displacement field is characterized by tracking the position of small particles randomly distributed on the surface membrane. Besides, the amplitude and the length of wrinkles are measured with a simple procedure based on the deflection of a lattice of equidistant parallel lines projected onto the membrane. Both methods are described in detail in the experimental section. In Section 3, in addition to measurements of the length and the amplitude of wrinkles, we test the number of wrinkles by varying the size of the pulling circle, the pulling central force and the membrane tension. In Section 4 we introduce the basis of our theoretical model and we contrast it with our experimental results. For the sake of continuity the complete description of our semi-analytical calculations is developed in the appendix. Finally, our main conclusions are presented in Section 5.

\section{Problem geometry}

\subsection{Definitions}

Aiming to mimic the contraction of a cell attached to a stretched membrane, we consider the geometry sketched in Figure 1. The circular membrane is pulled toward the center along a circle (radius $\left.r_{0}\right)$. We denote $u_{r}\left(r_{0}\right)=-\delta$ the imposed radial displacement. In order to account for the initial stretching of the membrane, we impose the displacement $u_{r}(R)=\beta$ along the large outer radius, $R$. In the following, we denote $\mathbf{u}(r, \theta)$ the horizontal-displacement field and $\zeta(r, \theta)$ the out-of-plane displacement of the membrane. The general set of equations governing the equilibrium shape of the membrane is given in the appendix.

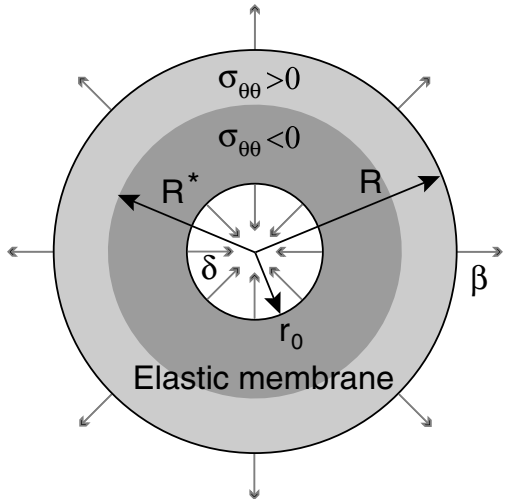

Fig. 1. Sketch of the experimental configuration. The main quantities defined in the text are indicated in the figure. The darker section, limited by $R^{*}$, indicates the membrane region, under azimuthal compression, where wrinkling might occur.

\subsection{Axi-symmetrically stretched membrane}

If the mechanical situation is stable with respect to the buckling of the membrane $(\zeta=0)$, taking into account the imposed deformation, one shows easily that the horizontal-displacement field reduces to $u_{r}$, which obeys $\Delta u_{r}=0$. Taking into account the boundary conditions, one obtains

$$
u_{r}(r)=\frac{\delta r_{0}+\beta R}{R^{2}-r_{0}^{2}} r-\frac{\delta R+\beta r_{0}}{R^{2}-r_{0}^{2}} \frac{R r_{0}}{r}
$$

and the corresponding components of the stress tensor

$$
\begin{aligned}
\sigma_{r r}= & \frac{E}{1-\sigma^{2}} \\
& \times\left[(1+\sigma) \frac{\delta r_{0}+\beta R}{R^{2}-r_{0}^{2}}+(1-\sigma) \frac{\delta R+\beta r_{0}}{R^{2}-r_{0}^{2}} \frac{R r_{0}}{r^{2}}\right], \\
\sigma_{\theta \theta}= & \frac{E}{1-\sigma^{2}} \\
& \times\left[(1+\sigma) \frac{\delta r_{0}+\beta R}{R^{2}-r_{0}^{2}}-(1-\sigma) \frac{\delta R+\beta r_{0}}{R^{2}-r_{0}^{2}} \frac{R r_{0}}{r^{2}}\right], \\
\sigma_{r \theta}= & 0 .
\end{aligned}
$$

At this point, it is worth considering the case of a membrane stretched at the outer radius with the constant tension $\gamma$ without any constraint along the inner circle. One can easily obtain from equation (2) that the resulting displacement $\beta$ at the outer radius $R$ satisfies

$$
\gamma=\frac{E}{1-\sigma} \frac{\beta}{R}
$$

Note that the tension $\gamma$ is then associated to the initial displacements $\beta$ at the outer radius and $-\delta_{0} \equiv u_{r}\left(r_{0}\right)=$ $\beta r_{0} / R$ at the inner circle. Let us now impose an additional displacement $-\delta_{i}$ along the inner circle so that $\delta=\delta_{i}+$ $\delta_{0}$. Using equation (2), one can show that, in order to maintain the equilibrium, one must pull the membrane 
along the inner radius with the tension $\tau \equiv \sigma_{r r}\left(r_{0}\right)$ which implies

$$
\tau=E \frac{(1-\sigma) R^{2}+(1+\sigma) r_{0}^{2}}{\left(1-\sigma^{2}\right)\left(R^{2}-r_{0}^{2}\right)} \frac{\delta_{i}}{r_{0}}+\frac{\gamma}{1+\sigma} .
$$

Thus, there exists a linear relation between the imposed displacement $\delta_{i}$ and $\tau$, and the initial tension $\gamma$ is proportional to $\beta$. In the following, we will improperly use the "tensions" $\beta$ and $\delta_{i}$ (or $\delta$ ) instead of $\gamma$ and $\tau$.

Let us now analyze the behavior of the orthoradial stress $\sigma_{\theta \theta}$. Provided that $\delta>0$ and $\beta>0$, the radial component $\sigma_{r r}>0(\forall r)$, whereas $\sigma_{\theta \theta}$ changes in sign for the radius

$$
R^{*}=\sqrt{\frac{1-\sigma}{1+\sigma} \frac{\delta R+\beta r_{0}}{\delta r_{0}+\beta R} R r_{0}} .
$$

The orthoradial stress $\sigma_{\theta \theta}>0$ for $r>R^{*}$. In this region the membrane is stretched in both the directions of the $r$ - and $\theta$-axis. By contrast, $\sigma_{\theta \theta}<0$ for $r<R^{*}$. In this region, the membrane is subjected to a compression along the $\theta$-axis so that it is likely to buckle and to form wrinkles elongated along the $r$-axis. Let us now assume, in a crude approximation, that the buckling instability leads to the formation of $n$ radial wrinkles. The threshold of the instability at a distance $r$ from the center can be approximated by $\sigma_{\theta \theta}^{t} \propto E n^{2} h^{2} / r^{2}$, where $h$ denotes the thickness of the membrane. In the limit $R \rightarrow \infty$ and $r_{0} \delta \gg h^{2}$, one can guess that the membrane buckles as soon as $\sigma_{\theta \theta}<0$ so that the length of the wrinkles is of the order of $R^{*}$. In that limit

$$
R^{*}=r_{0} \sqrt{\frac{\tau-\frac{\gamma}{1+\sigma}}{\gamma}} .
$$

We recover here the result recently obtained by E. Cerda [10]. A good agreement was found between theory and experiment. The main conclusion is that the length of the wrinkles increases with the imposed tension $\tau$ and decreases when the initial tension of the membrane $\gamma$ is increased.

In addition, one can notice that, in the case of a membrane without any initial tension $(\beta=0)$, the radius $R^{*}=R \sqrt{(1-\sigma) /(1+\sigma)}$ is smaller than the outer radius $R$ of the membrane ( $\sigma>0$, in usual practical cases) and does not depend on the displacement $\delta_{i}$, imposed at the center. In this case, one can expect the characteristics of the wrinkles to be imposed mainly by the outer boundary, and, for instance, their length to be about the outer radius $R$.

Thus, when pulled at the center, the membrane is generally submitted to an orthoradial compression in a region, $r_{0}<r<R^{*}$, in which wrinkles elongated along the $r$-axis are likely to form. The result obtained above (Eq. (8)), is limited to the case of very thin membranes (or to large imposed displacements) and does not provide any information on the number of wrinkles $n$ nor on the wrinkle profile. In the following section, we describe the experimental realization of the proposed geometry and analyze the characteristics of the membrane instability close to the threshold of the instability.

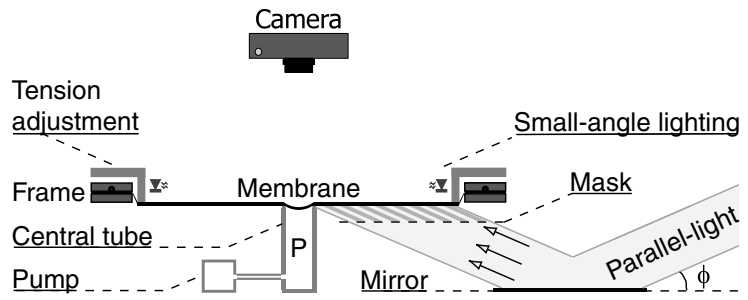

Fig. 2. Experimental setup.

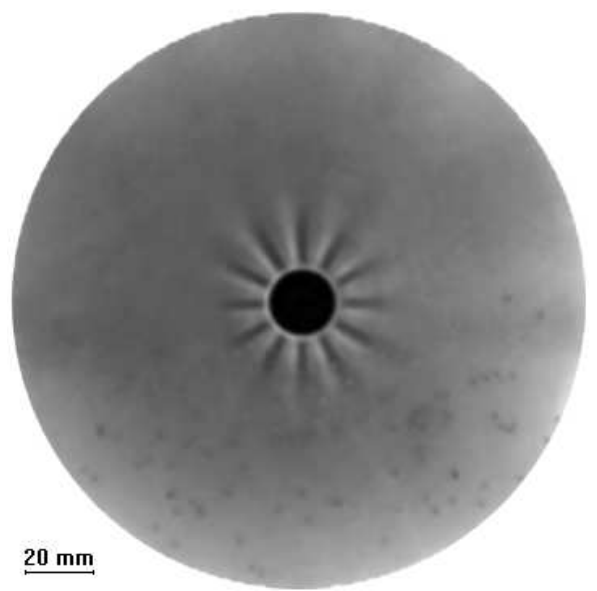

Fig. 3. Top view of the buckled membrane. Above a threshold displacement, radial wrinkles form around the central tube. One can clearly see them from above using the circular smallangle light source (12 wrinkles, $r_{0}=19 \mathrm{~mm}$ ).

\section{Experimental study}

\subsection{Experimental setup}

The experimental setup consists of a horizontal circular frame that holds firmly a thin elastic membrane (Latex, thickness $h=0.2 \mathrm{~mm}$ ) which is initially wedged between two plexiglass rings without any significant tension. The initial tension of the membrane, $\gamma$, can be adjusted later by pushing it down along a circle $(R=18.5 \mathrm{~cm})$ by means of a third L-shaped ring (Fig. 2).

One imposes the axi-symmetric radial displacement $\delta_{i}$ along a circle (radius $r_{0}$ ) by sucking in the membrane in a vertical tube (PVC); the inner pressure, $P$, is decreased with the help of a manual pump. The top circular edge of the tube is rounded and lubricated so as to allow the membrane to slide without any significant friction. A simple radial in-plane displacement over the whole surface of the stretched membrane results from the imposed deformation.

The system is imaged from above, along the vertical, with the help of a high-resolution digital camera (Nikon D1x). We use two different light sources; the membrane can be either lit from above by an annular array of LEDs, or from below by a parallel-light source. The upper light source (100 LEDs along a circle (radius $15 \mathrm{~cm}$ ), $1 \mathrm{~cm}$ above the upper surface of the membrane) provides a 


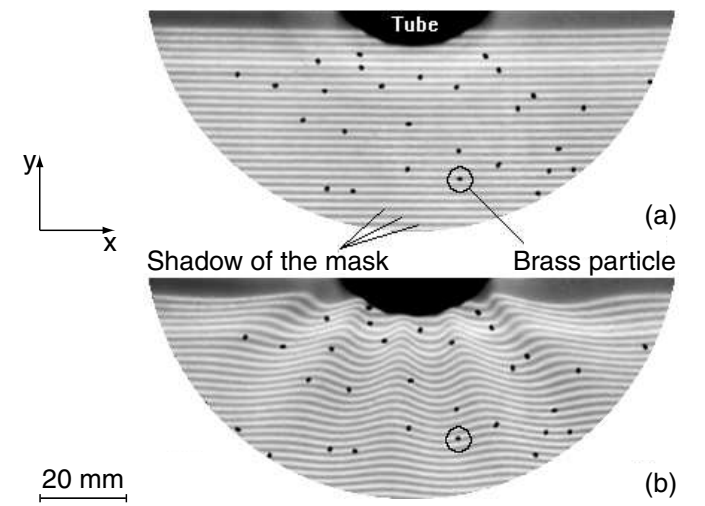

Fig. 4. Close-up view of the observed pattern. (a) For a flat membrane, the shadow of the mask corresponds to straight parallel lines along the $x$-axis. The brass particles, that are used to mark the in-plane displacement field, appear like black dots. (b) When the membrane is sucked in the central tube, the brass particles move toward the center, and the lines deform because of the vertical displacement of the membrane (12 wrinkles, $r_{0}=19 \mathrm{~mm}$ ).

homogeneous small-angle lighting which makes possible the direct observation of the out-of-plane membrane deformations. One can thus easily count the number of radial wrinkles that form around the central tube beyond the instability threshold (Fig. 3). The second light source is used for measuring the horizontal- and vertical-displacement fields; it consists of a parallel-light source casting the shadow of the mask (an array of lines parallel to the $x$ axis (Fig. 4)) onto the lower surface of the membrane, with the angle $\Phi$. The same images are used to determine both displacement fields as explained in the following.

In order to measure the in-plane displacement field, $\mathbf{u}(r)$, a large number of angular-shaped brass particles are randomly distributed onto the membrane (Fig. 4). Because of their angular shape, the brass particles cannot significantly roll over the surface. Thus, they mark the inplane displacement of the membrane, which is obtained by tracking all the particles positions between an image of reference $\left(\delta_{i}=0,(\right.$ Fig. $\left.4 \mathrm{a})\right)$ and the image of interest (Fig. 4b). The instability does not produce any significant ortho-radial displacement $u_{\theta}$, and the in-plane displacement field reduces to the radial component $u_{r}(r)$. The particles displacement, relative to the already stretched membrane $(\beta \neq 0)$, is given by

$$
\delta u_{r}(r)=-\delta_{i} \frac{r_{0}}{r} \frac{R^{2}-r^{2}}{R^{2}-r_{0}^{2}} .
$$

Interpolation of the experimental data to equation (9) allows us to determine accurately the imposed displacement $\delta_{i}$ within $0.1 \mathrm{~mm}$ (Fig. 5).

One obtains the local vertical displacement $\zeta(r, \theta)$ by analyzing the deformation of the pattern that the shadow of the mask forms on the membrane (Fig. 4). Let the $x$-axis be the axis parallel to the initially straight lines of the mask; the vertical-displacement field satisfies $\zeta(x, y)=-\tan \Phi \Delta y$, where $\Delta y$ denotes the local displacement of the pattern along the $y$-axis. The detection of the

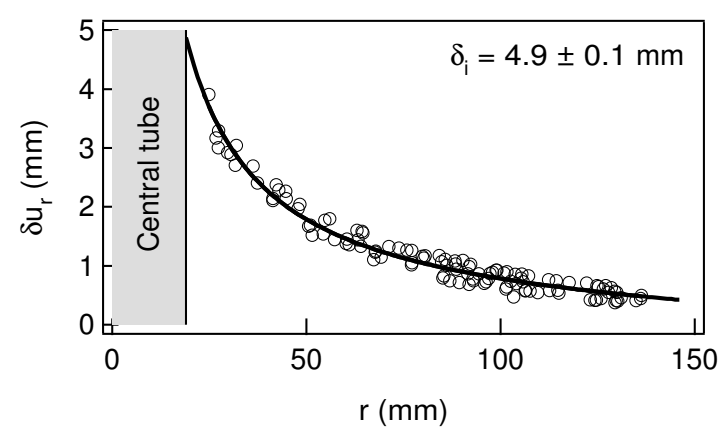

Fig. 5. Radial displacement $\delta u_{r}$ vs. radius $r$. Open circles: experimental data. Line: interpolation to equation (9) (from Fig. 4,115 brass particles detected, $r_{0}=19 \mathrm{~mm}, R=18.5 \mathrm{~cm}$, $h=0.2 \mathrm{~mm}$, and $\beta=2.4 \mathrm{~mm}$ ).

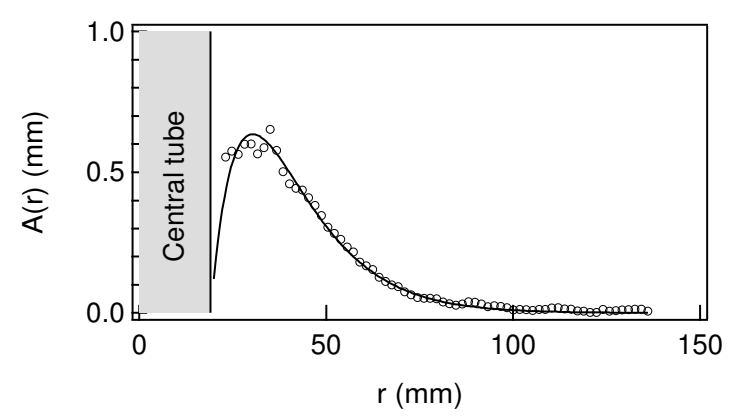

Fig. 6. Profile $A(r)$ vs. radius $r$. Open circles: experimental data. Line: guide to the eyes (from Fig. $4, \delta_{i}=4.9 \mathrm{~mm}$, $r_{0}=19 \mathrm{~mm}, R=18.5 \mathrm{~cm}, h=0.2 \mathrm{~mm}$, and $\beta=2.4 \mathrm{~mm}$ ).

bright and dark lines (Fig. 4), which is achieved by means of a data analysis software (Wavemetrics, Igor Pro 4), makes it possible to reconstruct the vertical-displacement field $\zeta(x, y)$ on one side of the central tube. The vertical displacement can be small. Thus, in order to achieve the required sensitivity, we assume that the verticaldisplacement field can be written $\zeta(r, \theta)=A(r) \sin (n \theta)$ and determine the profile $A(r)$. This procedure is accurate enough to detect the wrinkle amplitude $A(r)$ within $50 \mu \mathrm{m}$ in the range $r_{0}<r<140 \mathrm{~mm}$ (Fig. 6). Here, the number $n$ of wrinkles is obtained with the low-angle light source (see Fig. 3).

\subsection{Experimental results}

We determine the characteristics of the wrinkles as functions of the following experimental parameters; inner radius $r_{0}$, initial tension $\beta$ and imposed displacement $\delta_{i}$.

\subsubsection{Number of wrinkles $n$}

For given geometry (given $r_{0}$ and $R$ ) and initial tension of the membrane (given $\beta$ ), the number $n$ of wrinkles does not depend on $\delta_{i}$. On the other hand, $n$ increases linearly with the radius of the inner cylinder, $r_{0}$, while it tends to a finite value when $r_{0} \rightarrow 0$ (Fig. 7). In addition, $n$ increases slightly with the initial tension of the membrane $\beta$. 


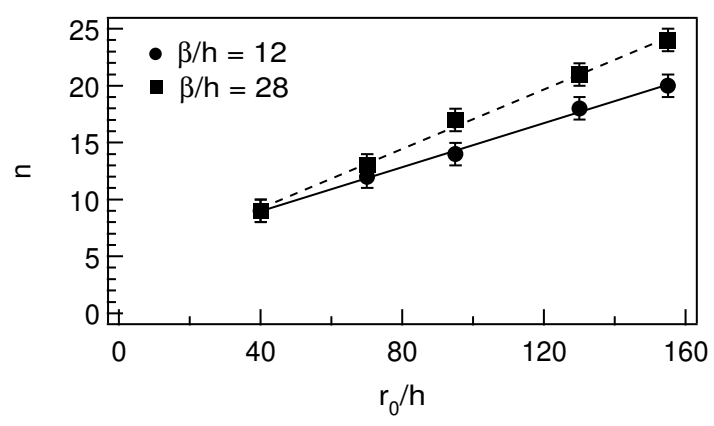

Fig. 7. Number $n$ vs. radius $r_{0}$. The number of wrinkles $n$ increases linearly with the radius of the inner cylinder $r_{0}$, while it tends to a finite value in the limit $r_{0} \rightarrow 0$ (typically 5 ). In addition, $n$ increases with the initial tension of the membrane $\beta(R=18.5 \mathrm{~cm}, h=0.2 \mathrm{~mm})$.

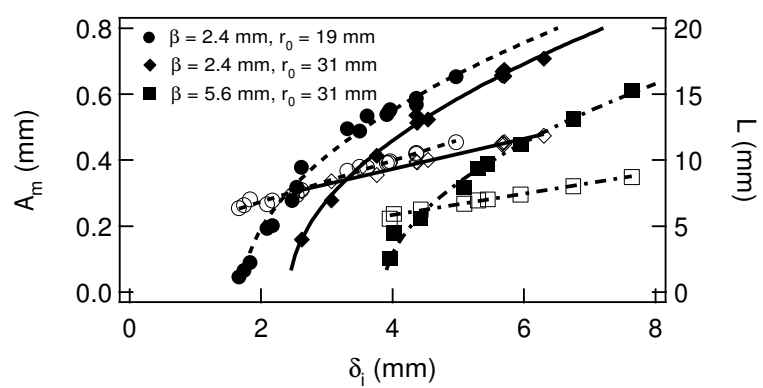

Fig. 8. Amplitude $A_{m}$ and length $L$ vs. $\delta$. Experimental measurements of the maximum amplitude $A_{m}$ and of the length $L$ as functions of the imposed displacement $\delta$. Full symbols: amplitude $A_{m}$; open symbols: length $L$. Lines: interpolation of $A_{m}$ to $A_{m}=\sqrt{K\left(\delta_{i}-\delta_{i}^{c}\right)}$ and of $L$ to $L=L_{c}+\xi \delta_{i}$. The results correspond to two different radii $r_{0}$ and two different values of the initial tension $\beta(R=18.5 \mathrm{~cm}, h=0.2 \mathrm{~mm})$.

\subsubsection{Amplitude $A_{m}$ and length $L$ vs. displacement $-\delta$}

From the experimental profiles $A(r)$ (Fig. 6), we determine the maximum amplitude $A_{m}$ and the typical length $L$ of the wrinkles, the latter defined to correspond to the inflexion point of the profile, $\mathrm{d}^{2} A / \mathrm{d} r^{2}\left(L+r_{0}\right)=0$. The wrinkles appear beyond a critical displacement $\delta_{i}^{c}$ (Fig. 8); the maximum amplitude $A_{m}$ satisfies $A_{m}=\sqrt{K\left(\delta_{i}-\delta_{i}^{c}\right)}$ whereas, starting from the finite value $L_{c}$, the length $L$ increases linearly with $\delta_{i}$. However, $L$ decreases when the radius $r_{0}$ and the tension $\beta$ are increased. The characteristics of the bifurcation will be discussed in details with the help of the numerical results in Section 4.3.2.

\subsection{Comments}

Even providing accurate measurements of the wrinkles characteristics, the experimental setup presents some limitations that deserve to be discussed at this point and that, in particular, justify the numerical analysis of the problem presented in Section 4.

The main limitation of the experimental setup consists of its inability to reach the limit $\beta \rightarrow 0$. Indeed, in this limit the weight of the membrane cannot be neglected anymore; experimentally, for small values of $\beta$, the membrane is not flat and the experimental setup fails to provide reliable measurements. Another limitation comes from the friction between the membrane and the central cylinder. We avoided this effect as much as possible but, at this point, we must prove that the characteristics of the wrinkles do not depend on the friction at this boundary. Finally, we cannot change easily the outer radius $R$ and the characteristics of the elastic membrane. We know, from the mathematical analysis, that the thickness $h$ of the membrane is the length scale, and that the results can be expressed in terms of $h$. In the same way, there is no need to know the elastic constant of the material as the characteristics of the wrinkles are only governed by geometrical relations. By contrast, we do not know accurately the value of the Poisson coefficient which is likely to play an important role (see, for instance, Eq. (7)). Moreover, this coefficient cannot be changed easily.

In the next section, we present numerical results that validate and extend our experimental findings.

\section{Numerical study}

\subsection{Set of equations}

Assuming that the membrane vertical deformation can be written in the form $\zeta(r, \theta)=A(r) \cos (n \theta)$, we show (see appendix) that the problem can be reduced to the following set of differential equations:

$$
\begin{aligned}
& r^{2} u_{r}^{0^{\prime \prime}}+r u_{r}^{0^{\prime}}-u_{r}^{0}=\frac{1}{4 r}\left[n^{2}(1+\sigma) A^{2}-2 n^{2} \sigma r A A^{\prime}\right. \\
& \left.\quad-(1-\sigma) r^{2} A^{\prime 2}-2 r^{3} A^{\prime} A^{\prime \prime}\right] \\
& \left(r^{4} A^{(4)}+2 r^{3} A^{(3)}\right) h^{2}-r^{2} A^{\prime \prime}\left[\left(1+2 n^{2}\right) h^{2}\right. \\
& \left.\quad+3\left(\sigma n^{2} A^{2}+r^{2} A^{\prime 2}\right)+12 r\left(\sigma u_{r}^{0}+r u_{r}^{0^{\prime}}\right)\right] \\
& +r A^{\prime}\left[\left(1+2 n^{2}\right) h^{2}-3 n^{2} A^{2}-12 r\left(u_{r}^{0}+\sigma r u_{r}^{0^{\prime}}\right)\right] \\
& \quad+n^{2} A\left[\left(n^{2}-4\right) h^{2}+12 r\left(u_{r}^{0}+\sigma r u_{r}^{0^{\prime}}\right)\right] \\
& +3 n^{4} A^{3}+3 n^{2} \sigma r^{2} A A^{\prime 2}-3 \sigma r^{3} A^{\prime 3}=0
\end{aligned}
$$

where $A(r)$ denotes the profile of the wrinkles along the $r$ axis and $u_{r}^{0}(r)$ the axi-symmetric part of the radial-displacement field. The solution to equations (10) and (11) can be found, provided the boundary conditions $u_{r}^{0}\left(r_{0}\right)=-\delta$ and $u_{r}^{0}(R)=\beta$ for the horizontal-displacement field $u_{r}^{0}(r)$, and $A\left(r_{0}\right)=0, A(R)=0, A^{\prime}\left(r_{0}\right)=0$, and $A^{\prime}(R)=0$ for the radial profile $A(r)$ of the wrinkles.

\subsection{Numerical method}

The coupled differential equations (10) and (11), associated to the corresponding boundary conditions, are solved 


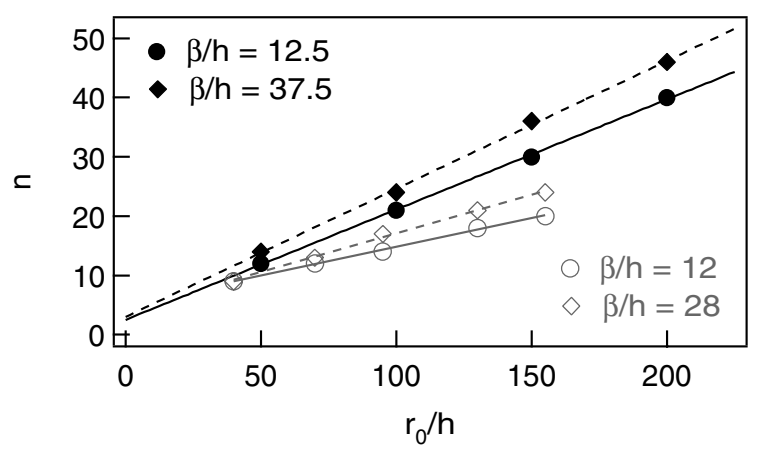

Fig. 9. Number $n$ vs. radius $r_{0}$. Full symbols: numerical data. Open symbols: experimental results from Figure 7. The number $n$ of wrinkles depends linearly on the radius $r_{0}$ of the central cylinder. The slope $\frac{\mathrm{d} n}{\mathrm{~d} r_{0}}$ increases with the initial tension of the membrane $\beta$. The number $n$ is found to tend to 2 or 3 when $r_{0} \rightarrow 0(\delta / h=50, R / h=1000$, and $\sigma=0.5)$.

numerically using a relaxation method. As the number of wrinkles, $n$, is a parameter in these equations, we compute initially the solutions for different values of $n$. Further analysis of the energy as a function of $n$ provides us with the number $n$ which minimizes the energy.

\subsection{Numerical results}

We compute the equilibrium shape of the membrane for values of the parameters similar to that of the experimental situation. In a first step, we choose a value of the Poisson coefficient, $\sigma=0.5$. The numerical displacement field $u_{r}^{0}$ and profile $A(r)$ are similar to the experimental ones presented in Figures 5 and 6 and are thus not reproduced in this section.

\subsubsection{Number of wrinkles $n$}

We compute the number of wrinkles, $n$, at equilibrium and observe that it depends only slightly on the imposed displacement $\delta$. By contrast, $n$ increases linearly with the radius $r_{0}$ of the central cylinder (Fig. 9). In agreement with the experimental results, $n$ is found not to vanish when $r_{0} \rightarrow 0$ and the slope $\frac{\mathrm{d} n}{\mathrm{~d} r_{0}}$ increases with the initial tension of the membrane $\beta$. However, we note that the experimental value of $n$ is always significantly smaller than that predicted numerically. The discrepancy remains unexplained but we note that, numerically, the energy difference between configurations corresponding to different values of $n$ is small. In our experimental configuration, the membrane is likely to be frozen by the effect of dissipation before reaching the optimal number of wrinkles. We can guess that an increase in the number of wrinkles would lead to enhanced dissipation as the deformation of the membrane would involve creation of more curvature. Thus, since we measured $n$ for increasing values of the tension, the observed number of wrinkles is smaller than the predicted one. Nevertheless, we note in the numerical results that the wrinkle profile $A(r)$ does not depend significantly on $n$ close to the energy minimum.
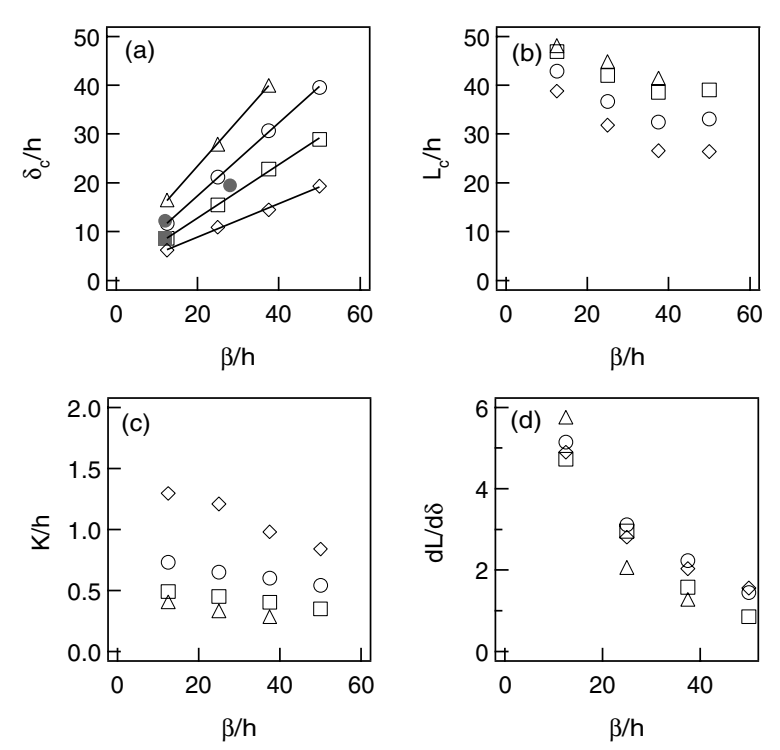

Fig. 10. Dimensionless $\delta_{c}, K, L_{c}$, and $\mathrm{d} L / \mathrm{d} \delta$ vs. $\beta$. The length of the wrinkles remains small enough compared to the outer radius for the system to be considered as infinite. Open symbols: numerical results (diamonds: $r_{0} / h=50$; circles: $r_{0} / h=100$; squares: $r_{0} / h=150$; triangles: $\left.r_{0} / h=200\right)$. Full symbols in (a): experimental data from Figure 8. $(R / h=1000$, and $\sigma=$ 0.5.)

\subsubsection{Instability threshold and bifurcation characteristics}

Again in agreement with the experimental results, the wrinkles are found numerically to appear beyond a critical value $\delta_{c}$ of the displacement $\delta$ which depends on the initial membrane tension $\beta$ (Fig. 10a). (In order to make comparison with the experiment easier, we report, in Fig. 10a, experimental results from Fig. 8. The discrepancy is always less than $20 \%$, better than the accuracy in $h$.) The numerical results show that $\delta_{c}$ increases linearly with $\beta$ and tends to a small but non-zero value (a few times the membrane thickness $h$ ) when $\beta$ vanishes. The length $L_{c}$ of the wrinkles at the threshold decreases with $\beta$ but increases with $r_{0}$ (Fig. 10b). In turn, above $\delta_{c}$, the maximum amplitude $A_{m}$ of the wrinkles increases like $A_{m}=\sqrt{K\left(\delta-\delta_{c}\right)}$, where the factor $K$ decreases (Fig. 10c) when the radius $r_{0}$ and the tension of the membrane $\beta$ are increased. The length of the wrinkles increases with $\delta$ and the slope $\mathrm{d} L / \mathrm{d} \delta$ decreases drastically when the tension of the membrane is increased. By contrast, we do not observe any appreciable variation of $\mathrm{d} L / \mathrm{d} \delta$ when the radius $r_{0}$ of the central cylinder is changed (Fig. 10d).

In Figure 11, we illustrate the explicit dependence of wrinkle amplitude and length on the main control parameter $\delta$. As pointed out above, the wrinkle amplitude follows a robust square root law as a function of $\delta$ (see lower panel of Fig. 11). The result holds true even in the limit $\beta=0$. However, the wrinkle length dependence on the pulling force is complex, depending strongly of membrane tension and the system size (see upper panel of Fig. 11). For small enough $\beta$, wrinkle length quickly saturates to the dimension of the system; $L$ tends asymptotically to 


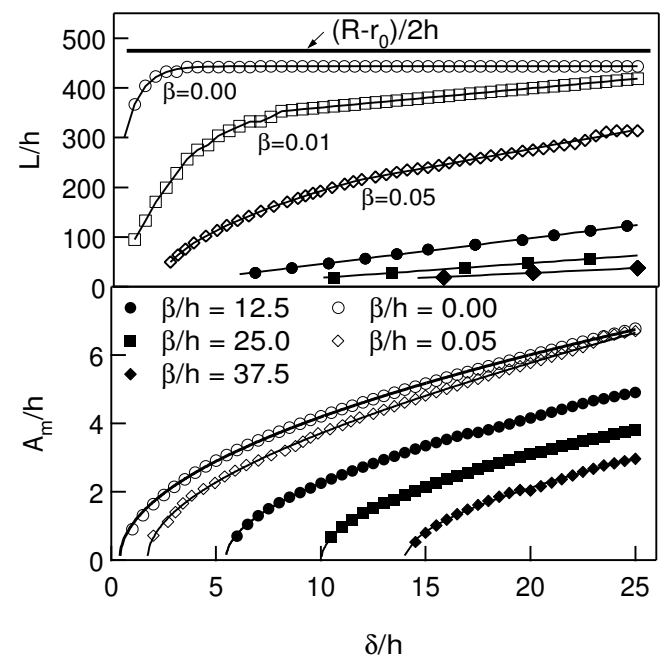

Fig. 11. Length $L$ and amplitude $A_{m}$ vs. $\delta$. The maximum amplitude $A_{m}$ of the wrinkles scales like $\sqrt{\delta-\delta_{c}}$ whatever the initial membrane tension $\beta$ (bottom). The length $L$ increases linearly with $\delta$ for large enough initial membrane tension $\beta$ $(\beta / h=12.5,25$ and 37.5 (full circles, squares, and diamonds, top)). If $\beta$ is small, departure from the linear law is observed close to the threshold $\delta_{c}(\beta=0,0.01$, and 0.05$)$. When $\beta=0$, the length $L$ of the wrinkles is rapidly limited by the dimension of the system. $\left(R / h=1000, r_{0} / h=50\right.$, and $\sigma=0.5$. $)$

roughly $\left(R-r_{0}\right) / 2 h$. At still small values of $\beta$, for which the system is large enough, we observe a nearly square root dependence of $L$ on $\delta$, corresponding to the regime reported recently in reference [10]. Notice that for small $\beta$ and $h$ nearly zero, $\delta_{c}$ is small and the square root dependence of $L$ extends to smaller values of $\delta$. However, as membrane tension $\beta$ is increased further, $L$ starts increasing linearly with $\delta$.

\subsubsection{Poisson ratio effects}

In this subsection we investigate how the results above are modified when the Poisson ratio is varied. In Figure 12a, we show the wrinkle profile for several values of $\sigma$. One can notice that for $\sigma=0$ the wrinkles extend up to the outer boundary. However, the wrinkle length decreases as $\sigma$ increases indicating that the Poisson effect opposes to buckling in this case. Thus, the main responsible of wrinkle formation is not the Poisson effect but instead a geometric azimuthal-contraction of the membrane due to the cylindrical geometry. Indeed, an axi-symmetric circular section of the membrane is naturally compressed when pulled toward the center. In turn, the number of wrinkles $n$ as a function of $\sigma$ is presented in Figure 12b. In Figure 12c, we show the maximum amplitude $A_{m}$ and length $L$ as functions of $\sigma$. $L$ decreases linearly when $\sigma$ is increased. The amplitude $A_{m}$ is nearly constant.
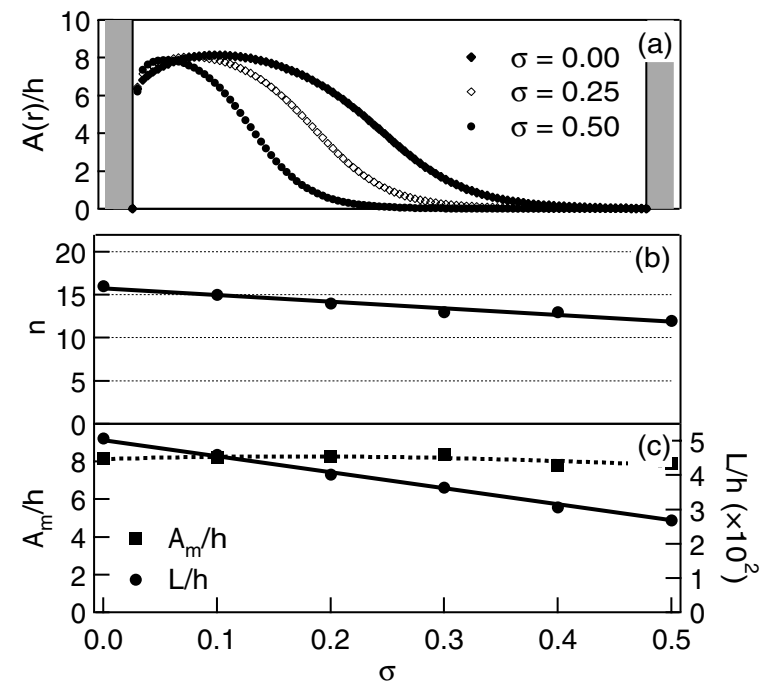

Fig. 12. Profiles $A(r)$, and $n, A_{m}$ and $L$ vs. $\sigma$. (a): Profile $A(r)$ for $\sigma=0.00,0.25$ and 0.50. For $\sigma=0.00$ the wrinkles extend up to the outer boundary, but they contract as $\sigma$ increases. (b): Number of wrinkles $n$ as a function of $\sigma$. (c): Maximum amplitude $A_{m}$ and length $L$ as functions of $\sigma .\left(r_{0} / h=50\right.$, $R / h=1000, \delta / h=50$, and $\beta / h=12.5$.)

\section{Conclusions}

In conclusion, to understand either the homogeneous contraction of a nearly circular living cell when attached to an elastic film or a circular wound, we have study in detail the geometry of a wrinkled membrane arising from axi-symmetric traction forces. We have shown that the wrinkle amplitude varies as the square root of the traction displacement, $A_{m} \sim \sqrt{\delta-\delta_{c}}$, beyond a well-defined critical value $\delta_{c}$, as in a supercritical instability. In turn, we show that wrinkle length increases linearly with traction displacement. However, in the limit of an infinitely thin and infinitely large membrane, we predict that wrinkle length goes like square root of tension, in agreement with recent independent results [10].

On the other hand, the number of wrinkles $n$, depends only slightly on the imposed displacements $\delta$ and $\beta$ but increases linearly with the radius $r_{0}$ of the central cylinder. At this stage one last question comes to mind and relates to what really selects the width of wrinkles, the distance from the center to the border on which membrane pulling occurs or simply its radius of curvature. To elucidate this question, the inner cylinder is now replaced by a square (edge $52 \mathrm{~mm}$ ) with rounded corners (radius of curvature $12.7 \mathrm{~mm}$ ). For a circle having the same radius of curvature, $r_{0}=12.7 \mathrm{~mm}$, one would expect $n=12\left(r_{0} / h \simeq 60\right.$, Fig. 7). Note that the characteristics of the wrinkles are similar to those observed in Figure 3. However, we do not observe any wrinkles in the flat parts of the inner frame, in agreement with the increase of $\delta_{c}$ with the radius of curvature of the boundary.

We now discuss some aspects of the technique introduced by Burton [12] to characterize membrane forces, produced by living cells, by measuring the wrinkle length. 


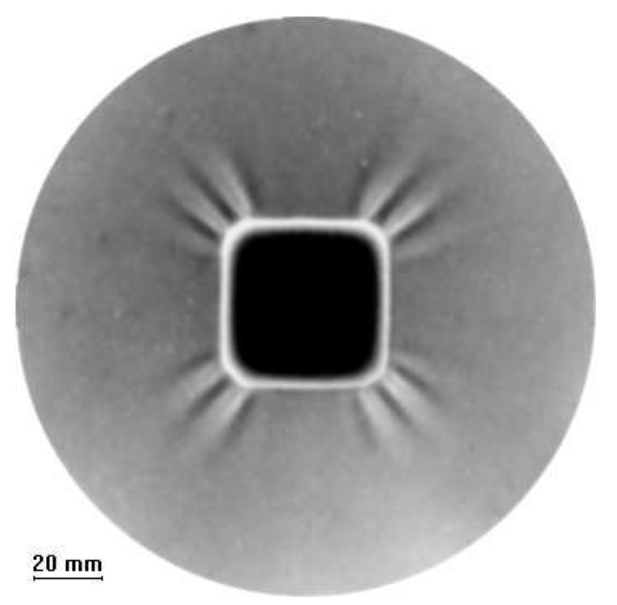

Fig. 13. Top view of the buckled membrane. The inner cylinder is replaced by a square (edge $52 \mathrm{~mm}$ ) with rounded corners (radius of curvature $12.7 \mathrm{~mm}$ ). The characteristics of the wrinkles on the round corners are similar to those observed in Figure 3. No wrinkles appear in the flat parts. $(R=18.5 \mathrm{~cm}$, $\beta / h=28$ and $h=0.2 \mathrm{~mm}$.)

First remark is that this method requires the wrinkle length to be defined and measured without any ambiguity. However, in experiments wrinkle length measurements are affected by large uncertainties. Here, we suggest to take wrinkle length as the distance from the pulling object to the inflexion point of the wrinkle profile. But, these measurements require precise knowledge of the wrinkle profile, information that is not currently available in such small systems. The second remark is that if the tension vanishes, our results predict that wrinkles develop in the whole membrane. This observation challenges the validity of force measurements since wrinkle length is no longer a function of the traction force. It might be argued that due to the preparation method of elastic membranes, see reference [12], there is always a membrane tension that limits the wrinkle length. However, to our knowledge, no independent measurements of such membrane tension have been carried out. Indeed, we believe that in case of vanishing membrane tension another mechanism controls the wrinkle length, for instance, gravity effects. In spite of the arguments above, if the wrinkle profile is available, then we suggest to measure the wrinkle maximum amplitude instead of the wrinkle length. This alternative method has more advantages. First, since the wrinkle amplitude is rather insensitive to Poisson effects, it does not require a precise knowledge of $\sigma$, a quantity which is hardly accessible in very thin membranes. Second and more important, beyond the instability threshold, wrinkle amplitude follows a robust square root law as a function of the pulling force. In contrast, wrinkle length dependence on the pulling force is complex, depending strongly on the membrane tension (see Fig. 11). Thus, we conclude that more reliable measurements to characterize forces acting on membranes are obtained with the wrinkle maximum amplitude.
Finally, let us mention that our calculations performed by using a simple semi-analytical method are in good agreement with experiments, providing a reliable tool to investigate configurations that are difficult to access experimentally. For instance, wrinkles in nano elastic films on finite-thickness viscous substrates used in semiconductor technology or cellular forces detection as described above. For these cases, our method can be generalized to include, for instance, gravity effect and viscous substrate.

We are very grateful to Fernando Lund and Enrique Cerda for many enlightening discussions and critical reading of the manuscript. This work was supported by Conicyt under Fondap Program No. 11980002. J.-C.G. thanks the Centre National de la Recherche Scientifique (France) for supporting the research of its members in foreign laboratories.

\section{Appendix A.}

In the present appendix, we show how the initial complex problem of minimizing the elastic energy with respect to the displacement fields $\mathbf{u}(r, \theta)$ and $\zeta(r, \theta)$ can be reduced to a set of two non-linear simple differential equations.

\section{Set of general equations}

Let us denote $\zeta(r, \theta)$ the scalar vertical-displacement field, and $u_{r}(r, \theta)$ and $u_{\theta}(r, \theta)$ the radial and ortho-radial components of the horizontal-displacement field $\mathbf{u}(r, \theta)$.

The elastic energy per unit surface associated to the bending deformation can be written [14] as

$$
\begin{aligned}
& E_{B}=\frac{E h^{3}}{24\left(1-\sigma^{2}\right)} \\
& \times\left[(\Delta \zeta)^{2}+2(1-\sigma)\right. \\
& \left.\times\left\{\left(\frac{1}{r^{2}} \frac{\partial \zeta}{\partial \theta}-\frac{1}{r} \frac{\partial^{2} \zeta}{\partial r \partial \theta}\right)^{2}-\left(\frac{\partial^{2} \zeta}{\partial r^{2}}\right)\left(\frac{1}{r} \frac{\partial \zeta}{\partial r}+\frac{1}{r^{2}} \frac{\partial^{2} \zeta}{\partial \theta^{2}}\right)\right\}\right]
\end{aligned}
$$

with

$$
\Delta \zeta=\frac{\partial^{2} \zeta}{\partial r^{2}}+\frac{1}{r} \frac{\partial \zeta}{\partial r}+\frac{1}{r^{2}} \frac{\partial^{2} \zeta}{\partial \theta^{2}},
$$

while the elastic energy per unit surface associated to the stretching deformation is given by

$$
\begin{aligned}
& E_{S}=\frac{E h}{2\left(1-\sigma^{2}\right)} \\
& \times\left[\left(u_{r r}+u_{\theta \theta}\right)^{2}+2(1-\sigma) \times\left(u_{r \theta}^{2}-u_{\theta \theta} u_{r r}\right)\right],
\end{aligned}
$$


where $u_{r r}, u_{r \theta}$ and $u_{\theta \theta}$ are the deformations:

$$
\begin{aligned}
& u_{r r}=\frac{\partial u_{r}}{\partial r}+\frac{1}{2}\left(\frac{\partial \zeta}{\partial r}\right)^{2}, \\
& u_{\theta \theta}=\frac{u_{r}}{r}+\frac{1}{r} \frac{\partial u_{\theta}}{\partial \theta}+\frac{1}{2 r^{2}}\left(\frac{\partial \zeta}{\partial \theta}\right)^{2}, \\
& u_{r \theta}=\frac{1}{2}\left(\frac{1}{r} \frac{\partial u_{r}}{\partial \theta}+\frac{\partial u_{\theta}}{\partial r}\right)-\frac{u_{\theta}}{2 r}+\frac{1}{2 r} \frac{\partial \zeta}{\partial r} \frac{\partial \zeta}{\partial \theta} .
\end{aligned}
$$

We remind that the stress tensor relates to the deformations according to

$$
\begin{aligned}
\sigma_{r r} & =\frac{E}{1-\sigma^{2}}\left(u_{r r}+\sigma u_{\theta \theta}\right), \\
\sigma_{\theta \theta} & =\frac{E}{1-\sigma^{2}}\left(\sigma u_{r r}+u_{\theta \theta}\right), \\
\sigma_{r \theta} & =\frac{E}{1+\sigma} \sigma u_{r \theta} .
\end{aligned}
$$

The equilibrium shape of the membrane minimizes the total energy $\iint\left(E_{S}+E_{B}\right) r \mathrm{~d} r \mathrm{~d} \theta$ with respect to the functions $\zeta(r, \theta), u_{r}(r, \theta)$ and $u_{\theta}(r, \theta)$.

\section{Buckling instability}

\section{Introduction}

Let us now assume that the stretched membrane is subjected to an instability that leads to the formation of $n$ identical wrinkles elongated along the $r$-axis. Helped by the experimental results, we write the verticaldisplacement field in the following form:

$$
\zeta(r, \theta)=A(r) \cos (n \theta)
$$

and solve the general set of equations in order to determine the number of wrinkles, $n$, and the profile, $A(r)$, that minimize the total elastic energy. The main problem is to determine the relations between the vertical- and horizontal-displacement fields $\zeta$ and $\mathbf{u}$. We show, in the following subsection, how the $n$-fold symmetry of the considered situation helps in overcoming this difficulty.

\section{Symmetries}

One can easily convince oneself that the displacement $u_{r}$ must be equal on both sides of a crest line (for instance, the radial line $\theta=0$ ) whereas the displacement $u_{\theta}$ must change in sign. Thus, $u_{r}$ and $u_{\theta}$ can be written as

$$
\begin{array}{r}
u_{r}(r, \theta)=u_{r}^{0}(r)+\bar{u}_{r}(r) \cos (2 n \theta), \\
u_{\theta}(r, \theta)=\bar{u}_{\theta}(r) \sin (2 n \theta) .
\end{array}
$$

The possible axi-symmetric deformation $u_{\theta}^{0}(r)$ is taken to be zero as we do not impose any twist of the membrane (or differential rotation of the boundaries).
In the same way, the component $\sigma_{r \theta}$ of the stress tensor must be odd in $\theta$, whereas $\sigma_{\theta r}$ must be even in $\theta$. The stress tensor being symmetric, these two conditions impose $\sigma_{r \theta}=\sigma_{\theta r}=0$, and, from equation (A.9), $u_{r \theta}=0$. Then, replacing $\zeta, u_{r}$, and $u_{\theta}$ by their expressions (A.10), (A.11), and (A.12) in equation (A.6) leads to

$$
r \bar{u}_{\theta}^{\prime}-\bar{u}_{\theta}-2 n \bar{u}_{r}=\frac{1}{2} n A A^{\prime} .
$$

Moreover, $\sigma_{r r}$ must be even and $\sigma_{\theta \theta}$ odd in $\theta$, so that these functions can be written as

$$
\begin{aligned}
& \sigma_{r r}(r, \theta)=\sigma_{r r}^{0}(r)+\bar{\sigma}_{r r}(r) \cos (2 n \theta), \\
& \sigma_{\theta \theta}(r, \theta)=\sigma_{\theta \theta}^{0}(r)+\bar{\sigma}_{\theta \theta}(r) \sin (2 n \theta) .
\end{aligned}
$$

Introducing the expressions (A.10), (A.11), and (A.12) in the general relations (A.7) and (A.8), and using the relations (A.14) and (A.15), one obtains the following important relations between the stress tensor and the displacement fields:

$$
\begin{gathered}
\left(1-\sigma^{2}\right) \sigma_{r r}^{0}=u_{r}^{0^{\prime}}+\sigma \frac{u_{r}^{0}}{r}+\frac{\sigma n^{2}}{4 r^{2}} A^{2}+\frac{1}{4} A^{\prime 2}, \\
\left(1-\sigma^{2}\right) \sigma_{\theta \theta}^{0}=\sigma u_{r}^{0^{\prime}}+\frac{u_{r}^{0}}{r}+\frac{n^{2}}{4 r^{2}} A^{2}+\frac{\sigma}{4} A^{\prime 2} \\
\bar{\sigma}_{r r}=\bar{u}_{r}^{\prime}+\frac{1}{4} A^{\prime 2}=-\frac{1}{\sigma}\left(2 n \frac{\bar{u}_{\theta}}{r}+\frac{\bar{u}_{r}}{r}-\frac{n^{2}}{4 r^{2}} A^{2}\right), \\
\bar{\sigma}_{\theta \theta}=0 .
\end{gathered}
$$

Note that equations (A.13) and (A.18) make it possible to write the derivatives $\bar{u}_{r}{ }^{\prime}$ and $\bar{u}_{\theta}{ }^{\prime}$ as functions of $\bar{u}_{r}$ and $\bar{u}_{\theta}$ and of the derivatives of the amplitude $A$.

\section{Minimization of the elastic energy}

The equilibrium shape of the membrane minimizes the total elastic energy $\iint\left(E_{S}+E_{B}\right) r \mathrm{~d} r \mathrm{~d} \theta$. Thanks to equations (A.13) and (A.18), the energy per unit area $\left(E_{S}+\right.$ $\left.E_{B}\right)$ can be rewritten as a function of $\bar{u}_{r}(r)$ and $\bar{u}_{\theta}(r)$ without any occurence of their derivatives. Thus, the minimization of the total energy with respect to these two functions reduces to $\frac{\mathrm{d} r\left(E_{S}+E_{B}\right)}{\mathrm{d} \bar{u}_{r}}=0$ and $\frac{\mathrm{d} r\left(E_{S}+E_{B}\right)}{\mathrm{d} \bar{u}_{\theta}}=0$ and leads to the unique relation:

$$
r \bar{u}_{r}+\frac{n}{2} r \bar{u}_{\theta}=\frac{n^{2}}{4} A^{2} .
$$

Introducing this last relation in $E_{S}$ (Eq. (A.3)) allows us to rewrite the total energy as a function of $u_{r}^{0}(r)$ and $A(r)$ only.

The minimization of the total elastic energy with respect to $u_{r}^{0}(r)$ consists in writing

$$
\frac{\mathrm{d} r\left(E_{S}+E_{B}\right)}{\mathrm{d} \bar{u}_{r}^{0}}-\frac{\mathrm{d}}{\mathrm{d} r}\left[\frac{\mathrm{d} r\left(E_{S}+E_{B}\right)}{\mathrm{d} \bar{u}_{r}^{0}}\right]=0,
$$

that leads to equation (10) of Section 4.2. In the same way, the minimization with respect to $A(r)$ leads to equation (11). 


\section{References}

1. S.P. Timoshenko, J.M. Gere, Theory of Elastic Stability (McGraw Hill, New York, 1988).

2. See, for instance, B. Roman, A. Pocheau, Europhys. Lett. 46, 602 (1999); B. Audoly, B. Roman, A. Pocheau, Eur. Phys. J. B 27, 7 (2002)

3. E. Cerda, K. Ravi-Chandar, L. Mahadevan, Nature 419, 579 (2002).

4. E. Cerda, L. Mahadevan, Phys. Rev. Lett. 90, 1 (2003).

5. N. Sridhar, D.J. Srolovitz, Z. Suo, Appl. Phys. Lett. 75, 2482 (2001).

6. H.Z. Yin, R. Huang, K.D. Hobart et al., J. Appl. Phys. 94, 6875 (2003).
7. See, for instance, K.D. Hobart, F.J. Kub, M. Fatemi, M.E. Twigg, P.E. Thompson, T.S. Kuan, C.K. Inoki, J. Electron. Mater. 29, 897 (2000).

8. K.D. Hobart, 2001 International Conference on Alternative Substrate Technology, J. Electron. Mater. 30, 797 (2001).

9. S.P. Lacour, S. Wagner, Z.Y. Huang et al., Appl. Phys. Lett. 82, 2404 (2003).

10. E. Cerda, Mechanics of scars, to be published in J. Biomech. (2004).

11. A.K. Harris, P. Wild, D. Stopak, Science 208, 177 (1980).

12. K. Burton, D.L. Taylor, Nature 393, 150 (1998).

13. R. Bernal, J.-Ch. Géminard, F. Melo, in preparation.

14. L.D. Landau, E. M Lifchitz, Theory of Elasticity (Pergamon, New York, 1959). 